\title{
Research Paper \\ Comparison the Effect of Eight Weeks Pyramid Resistance Training With Two Patterns on Bio-motor Ability and Anthropometrical in Wrestlers
}

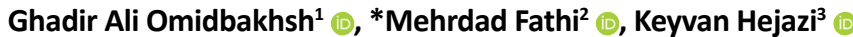

1. Department of Exercise Physiology, Faculty of Humanities, Bojnourd Branch, Islamic Azad University, Bojnourd, Iran.

2. Department of Exercise Physiology, Faculty of Sport Sciences, Ferdowsi University of Mashhad, Mashhad, Iran.

3. Department of Exercises Physiology, Faculty of Sport Sciences, Hakim Sabzevari University, Sabzevar, Iran.

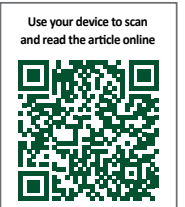

Crtation: Omidbakhsh GA, Fathi M, Hejazi K. [Comparison the Effect of Eight Weeks Pyramid Resistance Training With Two Patterns on Bio-motor Ability and Anthropometrical in Wrestlers (Persian)]. Journal of Sport Biomechanics. 2020; 6(2):110-121. https://doi.org/10.32598/biomechanics.6.2.3

doi'https://doi.org/10.32598/biomechanics.6.2.3

Keywords:

Resistance training, Physical fitness, Body Mass Index

\section{A B STRACT}

Objective Wrestling is a fast-paced activity in which resistance training is essential to improve athlete performance. We evaluated the effect of comparison of the effect of eight weeks resistance training with two patterns of pyramidal and inverse pyramidal on bio-motor ability, Anthropometrical and skill profile of freestyle wrestlers.

Methods In this semi experimental study, 24 male freestyle wrestlers were randomly assigned into two groups [pyramidal $(n=12)$ and inverse pyramidal endurance $(n=12)]$. The pyramidal and inverse pyramidal resistance training included ( 8 weeks, 3 times per a week, 60 minutes per session). Bio-motor ability, Anthropometrical and skill profile were measured at baseline and the end of the study. Paired and independent sample t-test were used to compare within and between-group means and the results were tested at the significant level $P<0.05$.

Results Training lead to changes on weight, body mass index, body fat percent, maximum power into pyramidal and inverse pyramidal groups $(\mathrm{P}<0.05)$. Anaerobic power, explosive power, muscular endurance increased significantly in both pyramidal and inverse pyramidal patterns. There were significant differences in the between-group mean changes in the variables of weight, body mass index and muscular endurance between the two experimental groups $(P<0.05)$.

Conclusion Resistance exercises with pyramidal and inverse pyramidal patterns led to significant weight loss, body mass index, body fat percentage, and improvement of skill profile and biometric indicators. Therefore, using both types of training may have a positive effect on wrestlers' performance indicators.

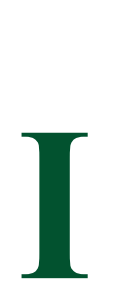

\section{Extended Abstract}

\section{Introduction}

n many sports, including wrestling, athletes need certain amounts of strength, depending on the type and nature of the skill performed and muscle utilization and muscles' role in optimal performance [2]. One of the best ways to increase muscle strength is to use increasing resistance training [3]. Physiological adaptations include increased strength, increased muscle size, and improved athletic performance [4]. There are various resistance training methods, among which we can mention the inverted and straightforward pyramidal methods [5]. These two training methods have been accepted as the most common weight training techniques for increasing strength and muscle hypertrophy [6].

\section{* Corresponding Author:}

Mehrdad Fathi, PhD.

Address: Department of Exercise Physiology, Faculty of Sport Sciences, Ferdowsi University of Mashhad, Mashhad, Iran

Tel: +98 (51) 38833910

E-mail: mfathei@um.ac.ir 
All wrestlers must have high levels of physical fitness and technical ability to succeed. Given that strength is one of the essential factors in wrestlers reaching the peak of readiness. On the other hand, wrestlers are afraid to deal with it due to the loss of technical skills, so some coaches and wrestlers believe that strength training has a detrimental effect (due to loss of flexibility, muscle stiffness, and reduced coordination in performing techniques) on improving and maintaining wrestlers' technical skills; therefore, coaches inhibit wrestlers from doing strength training. However, due to the importance of strength training in the process of success in wrestling competitions, wrestlers are always skeptical about doing these exercises and their detrimental effect on their skill profile and how to combine strength training with wrestling techniques.

The most important question for coaches and wrestling team members is resistance training with the intensity, volume, and duration that could be an excellent stimulus to increase muscle strength and positively affect skill profile. This study aimed to investigate the effect of eight weeks of resistance training with two pyramidal and inverse pyramidal patterns on bio-motor ability, anthropometric factors, and skill profile of freestyle wrestlers.

\section{Methods}

This research was a quasi-experimental study in which the two experimental and control groups were compared with pre-test and post-test design. The statistical population of this study included all male wrestlers in North Khorasan Province. They were members of the freestyle wrestling team of Bojnurd city, from which 24 people were selected voluntarily by available and purposive sampling. In the first stage, the subjects were familiarized with the project's nature and cooperated in its implementation. The samples were then randomly divided into simple pyramidal resistance training $(n=12)$ and reverse pyramidal resistance training $(n=12)$.

To evaluate the body composition of the subjects, their height was measured with a Seca gauge (with an accuracy of $5 \mathrm{~mm}$, made in Germany), their hip and waist circumference was measured with a MABIS tape measure (with a sensitivity of $5 \mathrm{~mm}$, made in Japan), and their body fat percentage and weight were measured using an In body-720 bioelectric impedance device (with a sensitivity of $100 \mathrm{~g}$, made in South Korea), respectively. The subjects' skill profile was measured using five techniques used in freestyle wrestling.
In this study, bio-motor indices including anaerobic power (sargent jump), explosive power (pair jump), muscular endurance (sit-up), and general agility (Illinois test) were collected. The Brzezinski formula was used to determine the 1RM (one-repetition maximum) of the subjects.

The training stations were as follows: the first station included a reciprocal superset of the forearm and back muscles; the second station included the reciprocal supersets of the quadriceps and hamstrings, and the third station included the reciprocal supersets of the chest and back muscles in two pyramidal and inverted pyramidal methods. The collected data were analyzed using SPSS software V. 16.

The Shapiro-Wilk heuristic statistical test confirmed the normality of the data's theoretical distribution, and Levene's test confirmed the homogeneity of the variances. Student's t-test and independent t-test were used to compare intra-group and inter-group means. A significance level of $\mathrm{P} \leq 0.05$ was considered for testing the results.

\section{Results}

The results of Table 1 prove that the changes of intragroup means in the variables of intra-group means (including body weight, BMI, body fat percentage, and maximum strength) were significantly changed in both inverse and straightforward pyramid patterns $(\mathrm{P}<0.05)$. The "skill profile" variable increased significantly only in the simple pyramid resistance training group. Changes in the inter-group mean in the "body mass index" variable were significantly different between the two experimental groups $(\mathrm{P}<0.05)$.

Changes in intra-group means in the variables of anaerobic power, explosive power, and muscular endurance increased significantly in both inverse and straightforward pyramidal patterns $(\mathrm{P}<0.05)$. The agility variable was significantly reduced in both groups of inverse and straightforward pyramidal resistance training. There was a significant difference between the two experimental groups in the variation of inter-group means in the muscle endurance variable $(\mathrm{P}<0.05)$.

\section{Discussion and Conclusion}

This study indicated that the BMI and body fat percentage decreased significantly in both inverted and straightforward pyramidal patterns. Simultaneously, anaerobic power, explosive power, muscular endur- 
Table 1. Comparison of the intra-group and inter-group variance changes in variables

\begin{tabular}{|c|c|c|c|c|c|c|c|}
\hline \multirow{3}{*}{ Variables } & \multirow{2}{*}{ Groups } & \multicolumn{2}{|c|}{ Mean \pm SD } & \multicolumn{4}{|c|}{ Changes } \\
\hline & & Pre-test & Post-test & \multicolumn{2}{|c|}{ Intra-group } & \multicolumn{2}{|c|}{ Inter-group } \\
\hline & \multicolumn{3}{|c|}{ Types of Resistance Training } & $\mathbf{P}$ & $\mathbf{t}$ & P* & $\mathbf{t}$ \\
\hline \multirow{3}{*}{ Weight (Kg) } & Simple pyramid & $71.83 \pm 0.93$ & $70.77 \pm 0.93$ & \multirow{3}{*}{0.04} & \multirow{3}{*}{-2.13} & 0.001 & 6.02 \\
\hline & & & & & & & \\
\hline & Inverse Pyramid & $71.33 \pm 1.43$ & $69.66 \pm 0.98$ & & & 0.001 & 7.41 \\
\hline \multirow{3}{*}{$\mathrm{BMI}\left(\mathrm{Kg} / \mathrm{m}^{2}\right)$} & Simple pyramid & $24.54 \pm 0.44$ & $24.18 \pm 0.39$ & \multirow{3}{*}{0.04} & \multirow{3}{*}{-2.11} & 0.001 & 5.99 \\
\hline & & & & & & & \\
\hline & Inverse Pyramid & $24.28 \pm 0.77$ & $23.71 \pm 0.67$ & & & 0.001 & 7.45 \\
\hline \multirow{3}{*}{ Body fat (Percentage) } & Simple pyramid & $19.4 \pm 2.7$ & $17.7 \pm 2.7$ & \multirow{3}{*}{0.71} & \multirow{3}{*}{-0.28} & 0.02 & 2.6 \\
\hline & & & & & & & \\
\hline & Inverse Pyramid & $19.5 \pm 4.9$ & $18.3 \pm 5.4$ & & & 0.04 & 2.3 \\
\hline \multirow{3}{*}{ Skill profile } & Simple pyramid & $79.2 \pm 9.6$ & $86.9 \pm 6.2$ & \multirow{3}{*}{0.37} & \multirow{3}{*}{0.9} & 0.04 & -3.3 \\
\hline & & & & & & & \\
\hline & Inverse Pyramid & $82.4 \pm 6.7$ & $83.9 \pm 7.2$ & & & 0.3 & -1.07 \\
\hline \multirow{2}{*}{$\begin{array}{c}\text { Muscle strength } \\
\text { (one-repetition maximum) }\end{array}$} & Simple pyramid & $311.6 \pm 58.9$ & $352.7 \pm 47.7$ & \multirow{2}{*}{0.88} & \multirow{2}{*}{0.14} & 0.02 & -4.9 \\
\hline & Inverse Pyramid & $304.5+48.4$ & $4216+1.40$ & & & 0.04 & -5.9 \\
\hline \multirow{3}{*}{$\begin{array}{l}\text { Anaerobic power } \\
\text { (centimeter) }\end{array}$} & Simple pyramid & $40.41 \pm 1.56$ & $41.33 \pm 1.30$ & \multirow{3}{*}{1.00} & \multirow{3}{*}{0.00} & 0.001 & -4.26 \\
\hline & & & & & & & \\
\hline & Inverse Pyramid & $39.58 \pm 1.31$ & $252.66 \pm 6.24$ & & & 0.02 & -2.68 \\
\hline \multirow{2}{*}{$\begin{array}{l}\text { Explosive power } \\
\text { (centimeter) }\end{array}$} & Simple pyramid & $245.75 \pm 6.57$ & $239.16 \pm 9.25$ & \multirow{2}{*}{0.60} & \multirow{2}{*}{-0.52} & 0.001 & -4.50 \\
\hline & Inverse Pyramid & $233.16 \pm 9.52$ & $65.91 \pm 1.67$ & & & 0.001 & -7.03 \\
\hline \multirow{3}{*}{$\begin{array}{l}\text { Muscular endurance } \\
\text { (Number) }\end{array}$} & Simple pyramid & $61.75 \pm 1.05$ & $66.91 \pm 2.23$ & \multirow{3}{*}{0.03} & \multirow{3}{*}{-2.25} & 0.001 & -8.01 \\
\hline & & & & & & & \\
\hline & Inverse Pyramid & $64.16 \pm 2.58$ & $8.91 \pm 0.26$ & & & 0.001 & -7.83 \\
\hline & Simple pyramid & $9.35 \pm 0.21$ & $8.75 \pm 0.36$ & & & 0.001 & 6.61 \\
\hline (Second) & & & & 0.62 & -0.49 & & \\
\hline & Inverse Pyramid & $9.25 \pm 0.20$ & $8.75 \pm 0.36$ & & & 0.002 & 4.19 \\
\hline
\end{tabular}

ance, and maximum power increased significantly in both inverted and straightforward pyramid patterns. Similar results in creating maximum strength and muscle volume in both reverse and straightforward pyramid training patterns are suitable for wrestling athletes. To this end, coaches are suggested that both training patterns can improve wrestlers' bio-motor ability indices.

\section{Ethical Considerations}

Compliance with ethical guidelines

All ethical principles were considered in this article. The participants were informed about the purpose of the research and its implementation stages; they were also assured about the confidentiality of their information; Moreover, They were allowed to leave the study whenever they wish, and if desired, the results of the research would be available to them.

Funding

This research did not receive any specific grant from funding agencies in the public, commercial, or not-forprofit sectors. 


\section{Authors' contributions}

All authors contributed equally in preparing all parts of the research.

\section{Conflicts of interest}

The authors declared no conflict of interest. 
This Page Intentionally Left Blank 


\title{
مقايسه اثر هشت هفته تمرين مقاومتى با دو الكّى باردهى هرمى بر شاخصهاى زيست حركتى وأنترويومتريك كثتى هثير هفته تمرين
}

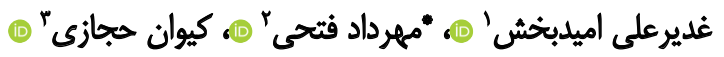 \\ ا.كروه فيزيولورثى ورزش، دانشكده علوم انسانى، واحد بجنورد، دانشَّاه آزاد اسلامى، بجنورد، ايران.

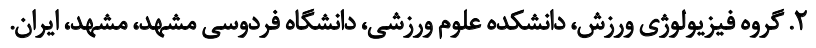

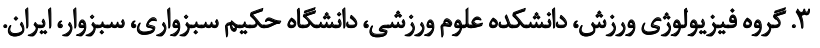

\begin{abstract}
حكند

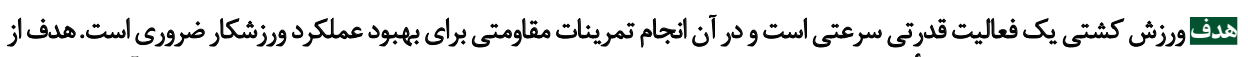

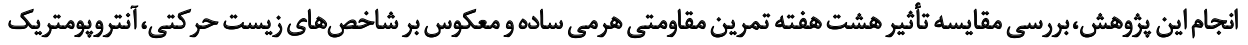
و ونيمرخ مهارتى كشتى ئيران آزادكار بودئ.

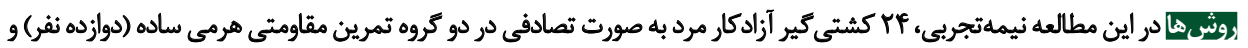

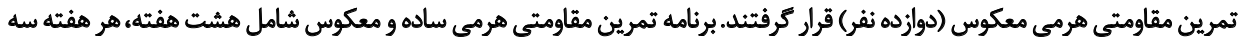

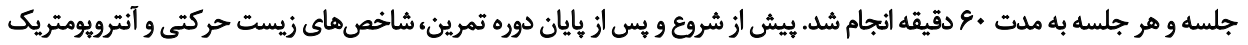

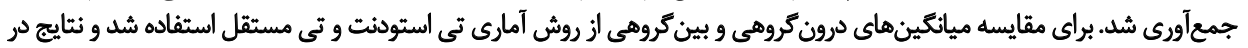

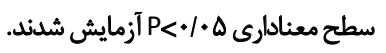

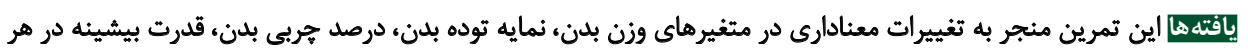

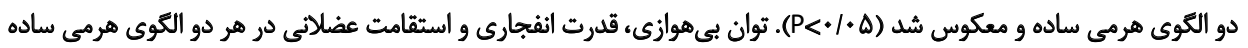

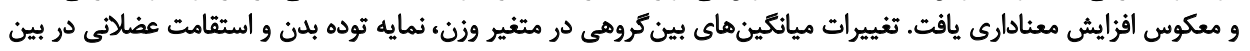

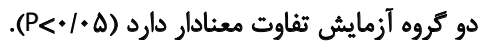

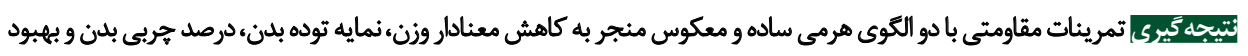

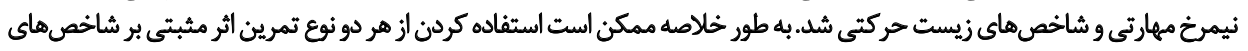
مملكردى كشتئي كيران داشتئه باشيد.
\end{abstract}

تاريخ دريافت: r T خرداد

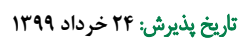

توان منقبض شود. تكرار اين نوع تمرينها به ايجاد سازكارىهاى

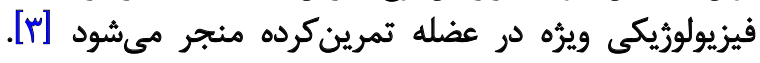

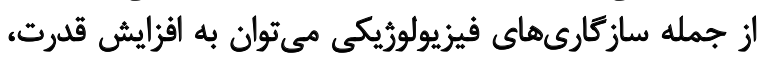

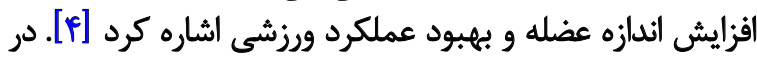

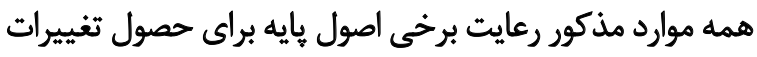

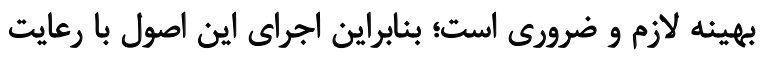

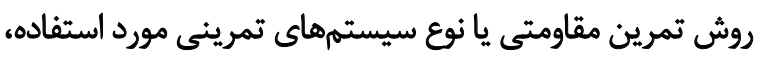

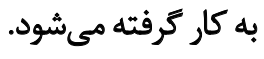

با تركيب مناسب برنامههاى مختلف تمرينى و دستكاري

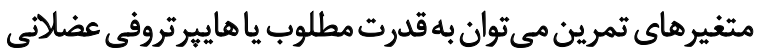

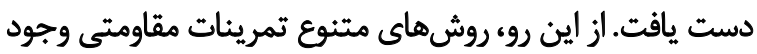

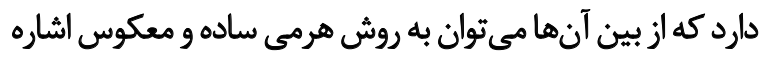

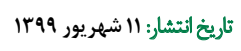

كليدواروها:

تمرين مقاومتى، آمادنى كائى

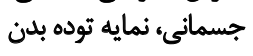

رشته كشتى يكى از باسابقهترين رشتههاى ورزشى است است بـان

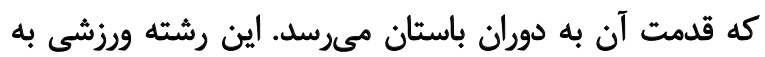

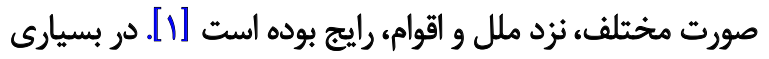

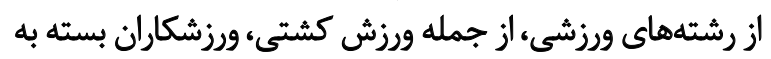

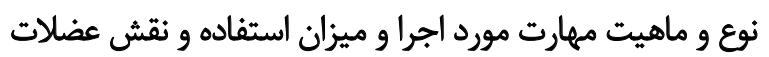

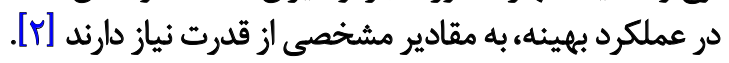
يكى از بهترين روشهاى افزايش قدرت عضلانى، استفاده از

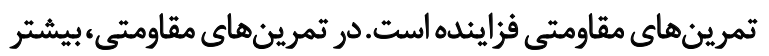

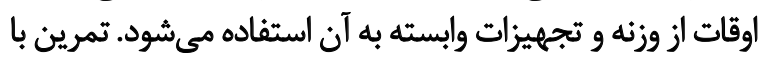
وزنههاي سنكين، عضله رادر وضعيتى قرار مي دهد كه به با بيشترين

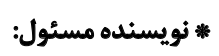

دكتر مهرداد فتحىى

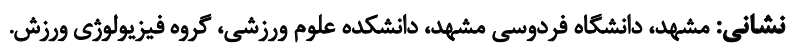

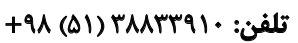
mfathei@um.ac.ir : يست الكترونيكي · 
كشتى گيران را از انجام تمرينات قدرتى منع مي كنيند و با توجه

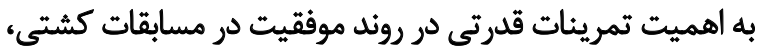

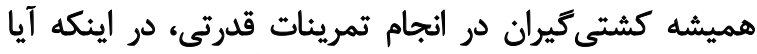

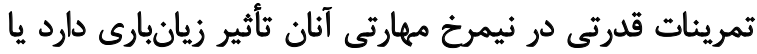

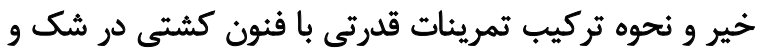
ترديل هستيند.

اهميت اين موضوع هنكامى دوجندان است كه حتى مربيان

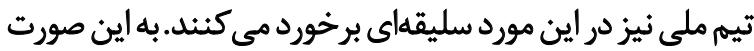

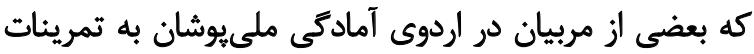

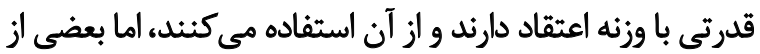

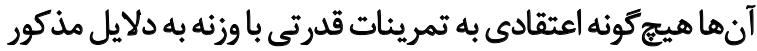

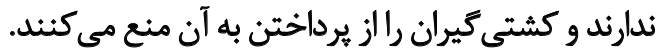
همجينين مهمترين سؤال براى مربيان و دستاندركاران

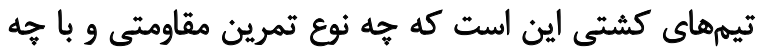

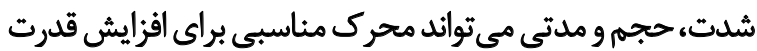

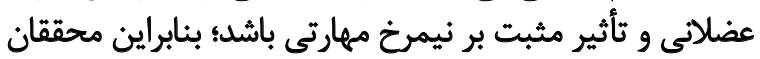

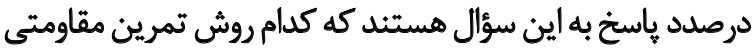

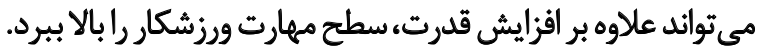

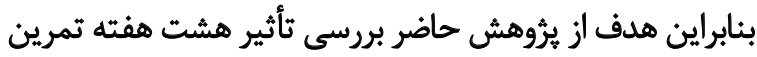

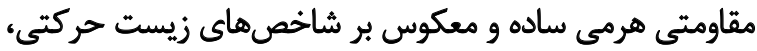

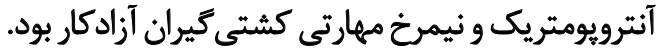

$$
\text { روش }
$$

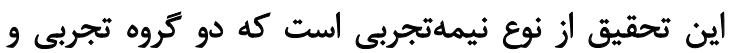

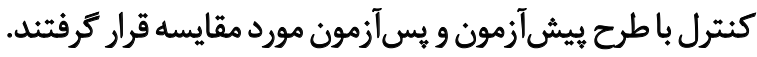

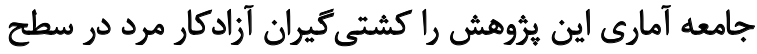

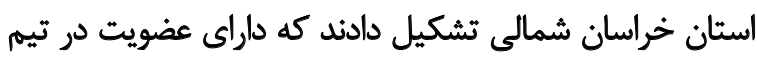

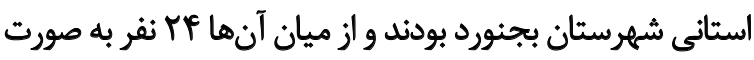

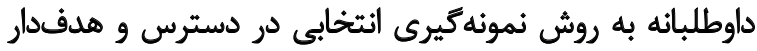

انتخاب شند.

در مرحله نخست افراد با ماهيت و نحوه همكارى با اجراي

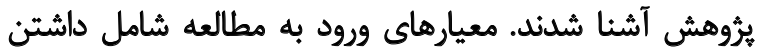

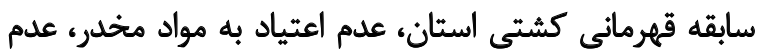

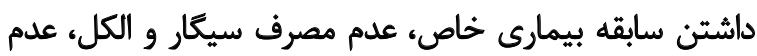

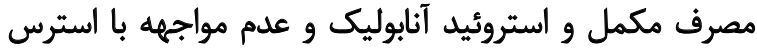

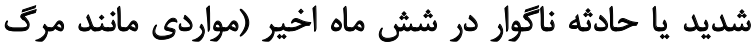

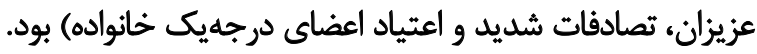

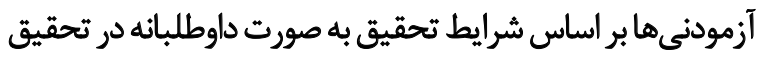

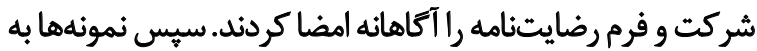

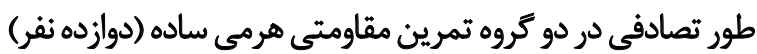

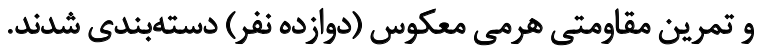

كرد [ه]]. اين دو روش تمرينى، به عنوان متداولترين روش هائ

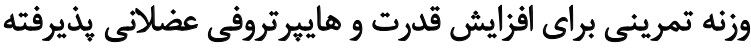

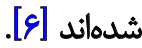

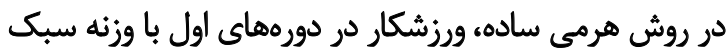

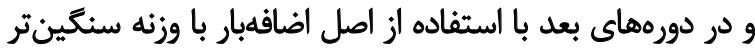

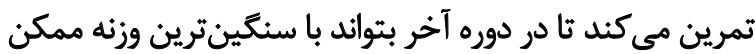

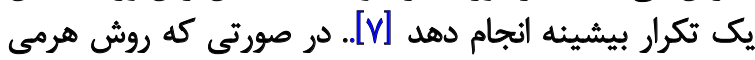

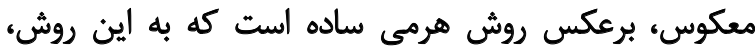

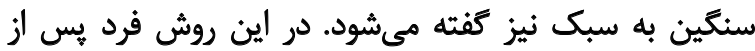

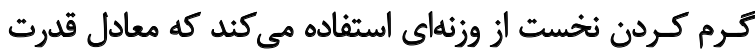

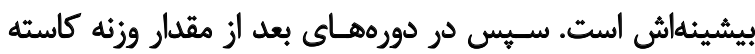

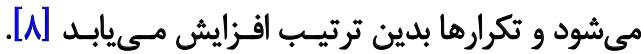

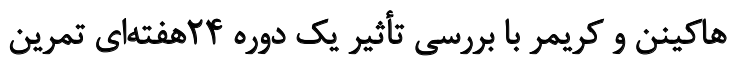

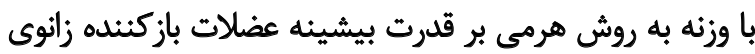

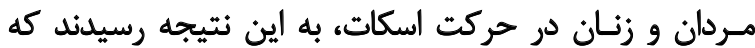

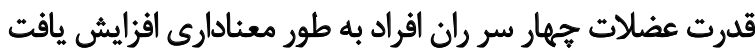

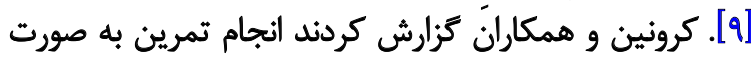

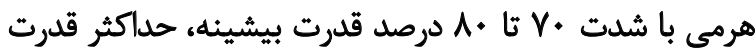

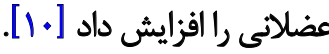

با توجه به اينكه تمرينات مقاومتى امروزه در بيشتر رشتههاى إئل

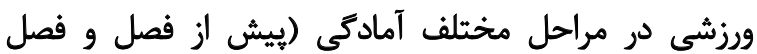

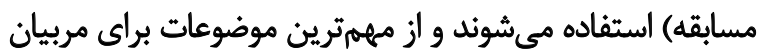

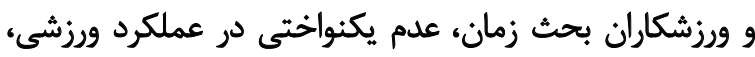

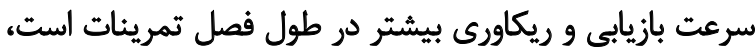

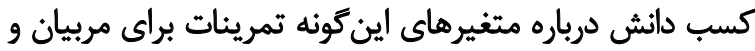

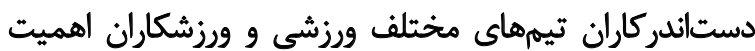

$$
\text { بسزايى دارد. }
$$

ورزش كشتى يك فعاليت قدرتى سرعتى است و در آن انجام

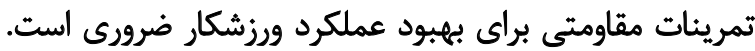

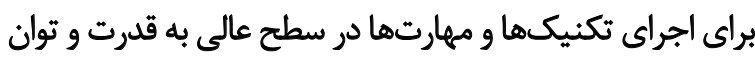

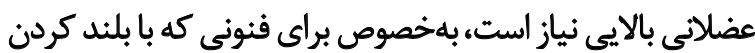
حريف همراه است [N].

تمامى كشتى كيران جهت كسب موفقيت بايستى سطوح

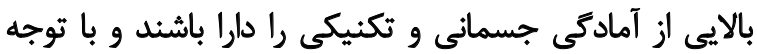

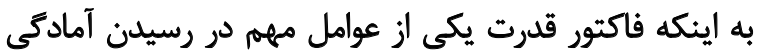

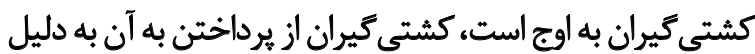
از دست دادن مهارتهان تكنيكى واهمه دارند.

در اين زمينه، شمارى از مربيان و كشتى كيران بر اين باورند

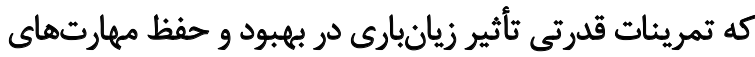

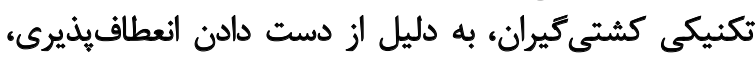

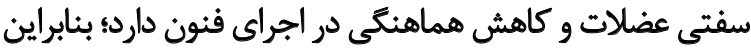


براى برآورد استقامت عضلانى از تست دراز و نشست استفاده

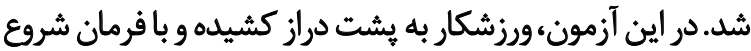

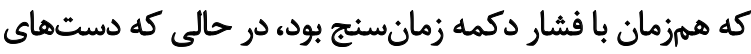

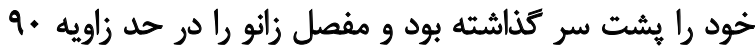

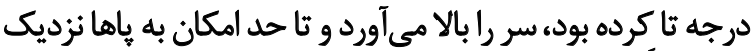

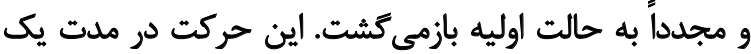

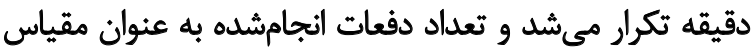

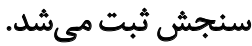

اندازمكيرى جابكى با آزمون ايلىنويز صورت يذيرفت. آزمودنى

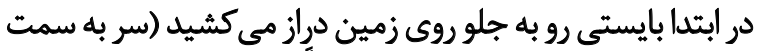

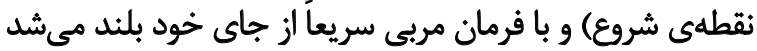

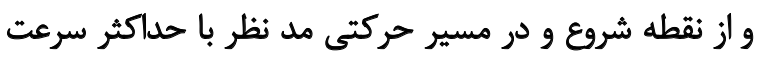

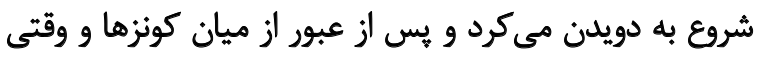

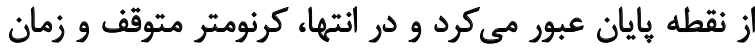

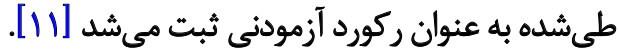
برنامه تمرين مقاومتى هرمى ساده و معكوس

براى تعيين قدرت يك تكرار بيشينه آزمودنىها از فرماني

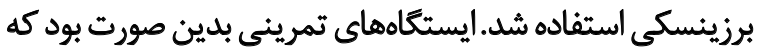

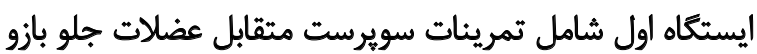

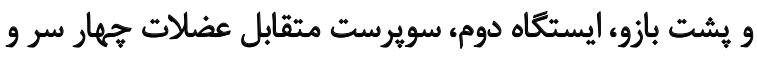

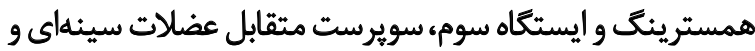
يشتى به دو روش هرمى ساده و معكوس بودئ

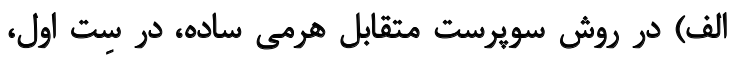

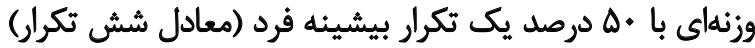

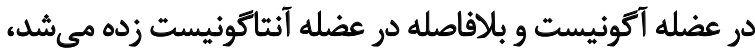

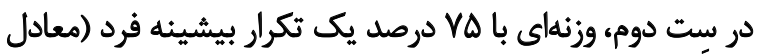

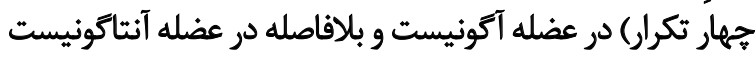

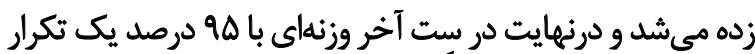

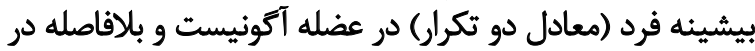
عضله آنتاكونيست زده مي (معادل دو تكرار در

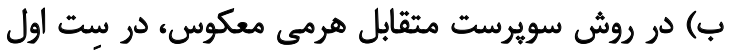

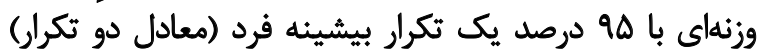

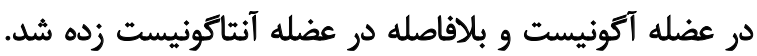

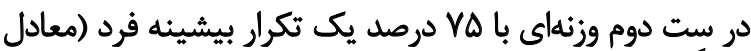

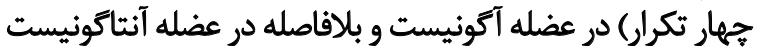

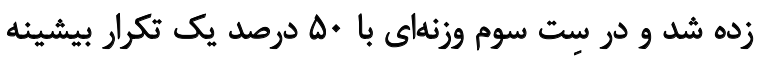

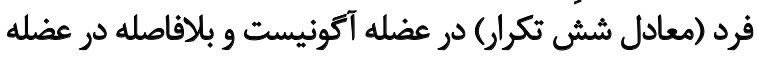

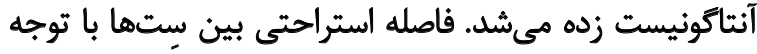

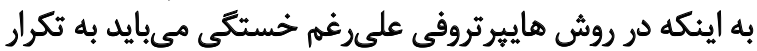

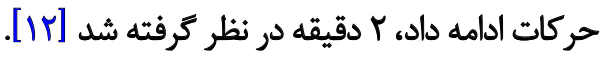

\section{تركيب بدن}

براى ارزيابى تركيبات بدن به ترتيب طول قد آزمودنىها با

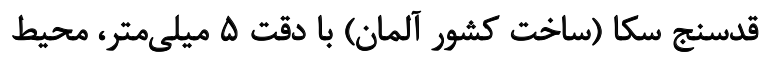

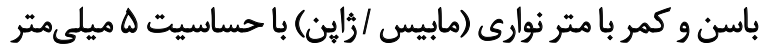

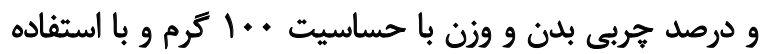

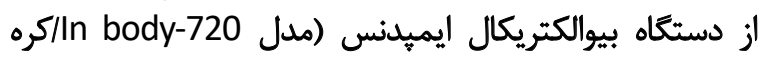

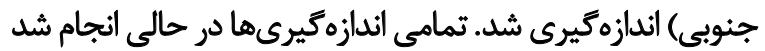

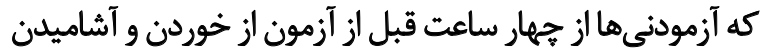

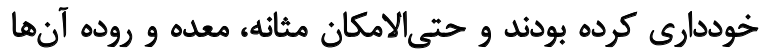

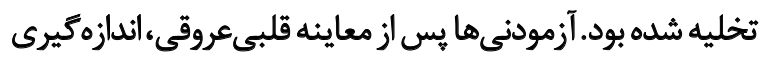

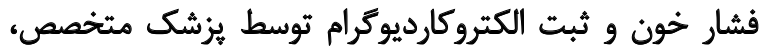
مجوز ورود به طرح را كسب كردند.

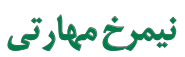

اندازمكيرى نيمرخ مهارتى با استفاده از ينج تكنيك مورد استفاده كشتى

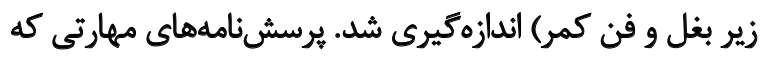

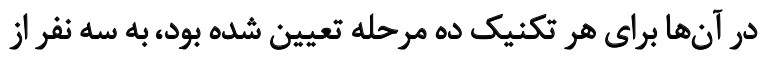

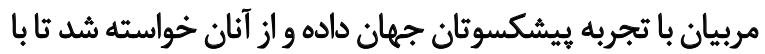

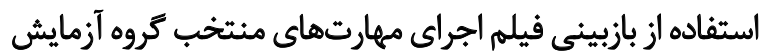
يك و دو بر اساس مقياس سهقيمتى ليكي ليكرت، آن را تكميل كنيند.

$$
\text { شاخص هاى زيست حركتى }
$$

در اين يُوهش شاخصهاى زيست حركنى شامل توان

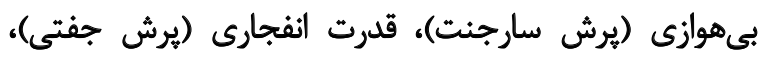

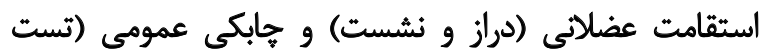

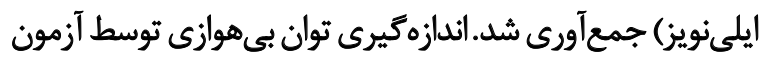

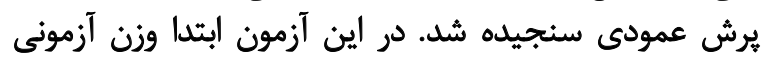

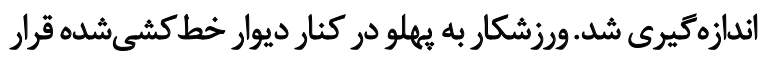

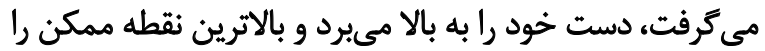

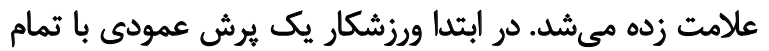

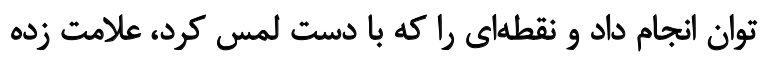

شويد.

فاصله بين دو نقطه علامتزدمشده نشاندهنده ميزان توان

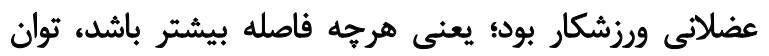

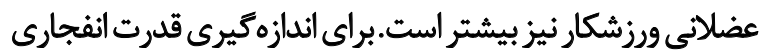

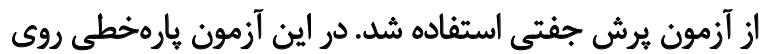

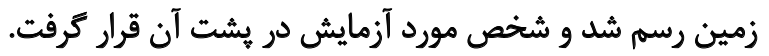

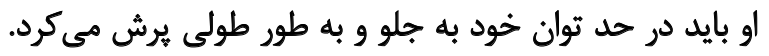

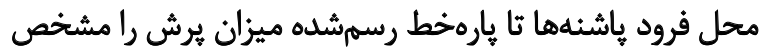

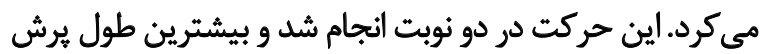

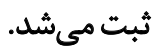




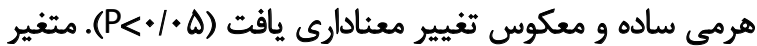

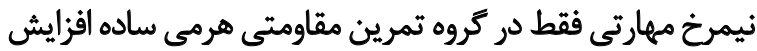

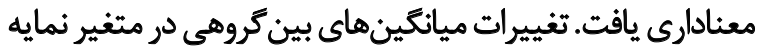

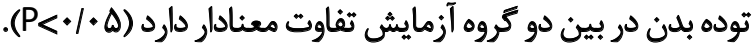
نتايج جدول شماره ب نشان مى دهد كه تغييرات ميانغينهاى

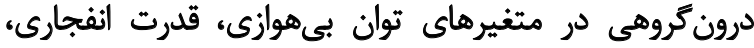

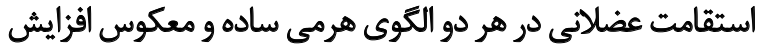

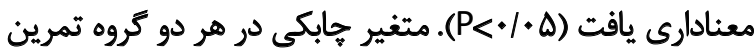

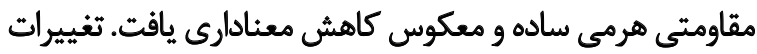

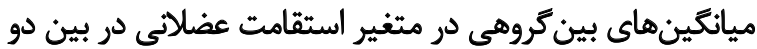

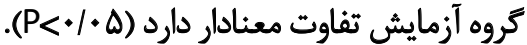

ثִ

هدف از مطالعه حاضر بررسى تأثير هشت هفته تمرين مقاومتى هاخي

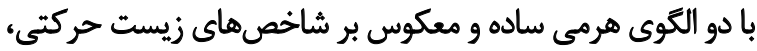

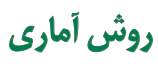

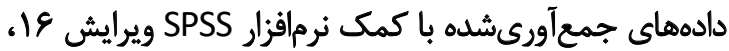

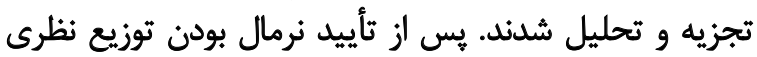

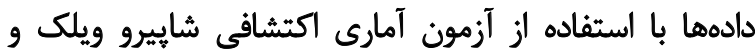

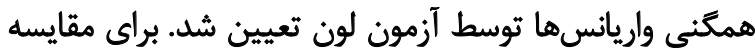

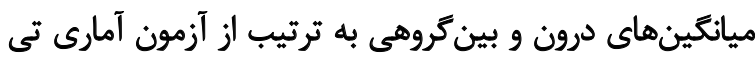

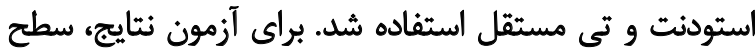

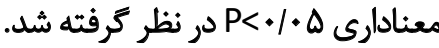

نئيج

مشخصات آزمودنىهاى كروه آزمايش و كنترل در جدان جدان

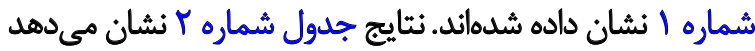

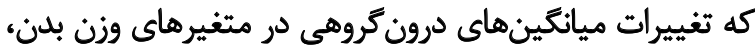

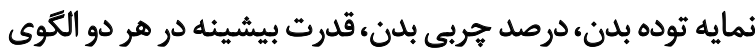

جدول ا. ويرُّى هاى آنترويومتريك شركت كنئله در مطالعه

\begin{tabular}{|c|c|c|c|c|}
\hline \multicolumn{4}{|c|}{ انحراف معيار ثميانكين } & \multirow{2}{*}{ 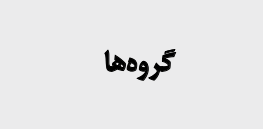 } \\
\hline نمايه توده بدن (كيلوكرم / مترمريع) & جرم (كيلوكرم) & قد (سانتىمتر) & سن (سال) & \\
\hline$M \varphi / \Delta F \pm=/ M \varphi$ & $V / A r \pm=/ q r$ & $|W| \cdot \lambda \pm 1 / \cdot 1$ & $r r / q \pm r / \varphi$ & هرمى ساده (دوازده نفر) \\
\hline TY/TAI./W & $V / r T \pm \pm / \pi r$ & $\mid V / P I \pm I / N$ & $r r / N \pm T / r$ & هرمى معكوس (دوازدده نفر) \\
\hline
\end{tabular}

مجله بيومكانيك وزنش

جدول r. مقايسه تغييرات واريانس درونكروهي و بين كروهي در شاخص هاى آنترويومتريك و نيمخ مهارتى كشتى كيران

\begin{tabular}{|c|c|c|c|c|c|c|c|}
\hline \multicolumn{4}{|c|}{ تغييرات } & \multicolumn{2}{|c|}{ ميانكين } & \multirow{3}{*}{ كروهاها } & \multirow{3}{*}{ متغيرها } \\
\hline \multicolumn{2}{|c|}{ يين كرووه } & \multicolumn{2}{|c|}{ ورون كروه } & \multirow{2}{*}{ بس آزمون } & \multirow{2}{*}{ بيش آزمون } & & \\
\hline $\mathbf{P}$ & $t$ & $\mathbf{P}$ & $\mathbf{t}$ & & & & \\
\hline \multirow{2}{*}{$+1+p=0$} & \multirow{2}{*}{$-r / I r$} & & & & $V / A r \pm+/ q u$ & هرمى ساده & \multirow{2}{*}{ (كيلوكرم) } \\
\hline & & $+1 \cdot+)^{*}$ & $V / F i$ & Eq/FEt./Q & VI/rTI $\pm 1 / 4 \pi$ & هرمى معكوس & \\
\hline \multirow{2}{*}{ 1.pen } & \multirow{2}{*}{$-r / I I$} & $+1+1^{*}$ & & & & & \multirow{2}{*}{ 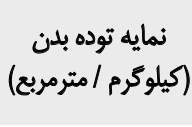 } \\
\hline & & $.1+.1^{*}$ & $V / F \Delta$ & $M T / M \pm \cdot / R V$ & rF/rAt./W & هرمى معكوس & \\
\hline \multirow{2}{*}{$\cdot M$} & \multirow{2}{*}{.$- / T A$} & & & $I V / V \pm r / V$ & $19 / 4 \pm T / Y$ & & \multirow{2}{*}{ درصد شرئ بلن } \\
\hline & &.$/+f^{*}$ & $r / T$ & $W^{N} \pm \Delta / f$ & $19 / \Delta \pm \varepsilon / 9$ & هرمى معكوس & \\
\hline \multirow{2}{*}{.$/ m V$} & \multirow{2}{*}{.19} &.$/ \cdot r^{*}$ & $-r / r$ & $N \varepsilon / q \pm \varepsilon / N$ & $V 9 / r \pm q / 8$ & هرمى ساده & \multirow{2}{*}{ نيمغ مهارتى } \\
\hline & & $\cdot / r$ & $-1 / \cdot v$ & $A r / q \pm V / r$ & $A r / F \pm g / V$ & هرمى معكوس & \\
\hline \multirow{2}{*}{$\cdot / M$} & \multirow{2}{*}{$\cdot / f$} & $.1 \cdot r^{*}$ & $-1 / 9$ & $r \Delta \& / \& \pm A Y / q$ & $M I / \varepsilon \pm \Delta N q$ & هرهي ساده & \multirow{2}{*}{ (يك تكرار بيشينه) } \\
\hline & & $.1+4$ & $-\Delta / 9$ & TAY/VETVN & $r+\varphi / \Delta \pm \Psi N F$ & هرمى معكوس & \\
\hline
\end{tabular}

مجله بيومكانيك ورنث

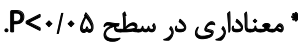


جدول سا. مقايسه تغييرات واريانس درون كروهى و بين كروهى در شاخصهاى زيست حركتى كشتى كيران

\begin{tabular}{|c|c|c|c|c|c|c|c|}
\hline \multicolumn{4}{|c|}{ تغييرات } & \multicolumn{2}{|c|}{ ميانكين +انحراف استاندارد } & \multirow{3}{*}{ كروهها } & \multirow{3}{*}{ متغيرها } \\
\hline \multicolumn{2}{|c|}{ بين كروه } & \multicolumn{2}{|c|}{ درون كروه } & \multirow{2}{*}{ يس آزمون } & \multirow{2}{*}{ يبش آزمون } & & \\
\hline $\mathbf{P}$ & $\mathbf{t}$ & $\mathbf{P}^{*}$ & $\mathbf{t}$ & & & & \\
\hline \multirow[t]{2}{*}{$1 / .0$} & \multirow[t]{2}{*}{.1.} & $.1 \cdot .1$ & -te/re & $\mu / / F \pm / / F$. & & & \multirow{2}{*}{ توان بى هوازيى } \\
\hline & & $\% H$ & -r/EA & FI/MTII/T. & $m q / \Delta A \pm 1 / m$ & هرهي معكوس & \\
\hline \multirow{2}{*}{+18} & \multirow{2}{*}{.$- / \Delta r$} & & $-F / Q$ & & & هرهى ساده & \multirow{2}{*}{ قدانتىمتر) } \\
\hline & & $.1+.1$ & $-V / \cdot r$ & $r \times q / / \& \pm q / r \Delta$ & mT/leta/ar & هرهي معكوس & \\
\hline \multirow{2}{*}{$+1+r^{2+*}$} & \multirow{2}{*}{$-r / T \Delta$} & $.1 . .1$ & $-N \cdot 1$ & $\varepsilon \Delta / q I \pm 1 / \varepsilon V$ & $8 / / V \Delta \pm 1 / \cdot \Delta$ & هرهي ساده & \multirow{2}{*}{ 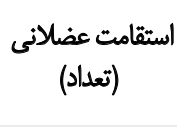 } \\
\hline & & $.1+.1$ & -V/Ar & $98 / 91 \pm r / r r$ & $E F / I E \pm Y / \Delta \Lambda$ & هرمى معكوس & \\
\hline \multirow{2}{*}{ - IEr } & \multirow[t]{2}{*}{$-. / 1 / q$} & $.1 . .1$ & $8|8|$ & NQI士./KE & $Q / T \Delta \pm \cdot(T)$ & هرمى ساده & \multirow{2}{*}{ (ثانيكي) } \\
\hline & & $\% r$ & $1 / 19$ & NVAI./YQ & $q / T \Delta \pm \cdot / T$. & هرئ معكوس & \\
\hline
\end{tabular}

بلافاصله يس از شروع فعاليت بدنى شديد، دستكاه كَليكوليز

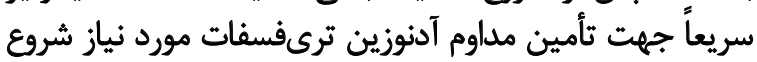

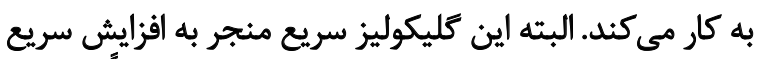

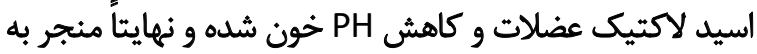

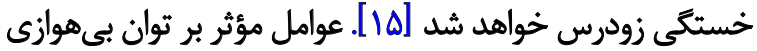

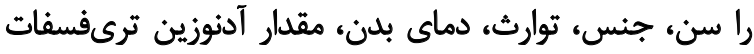

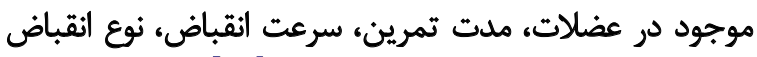

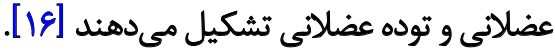
نتايج تحقيق حاضر نشان داد تمرينات مقاومتى با دو الكوى

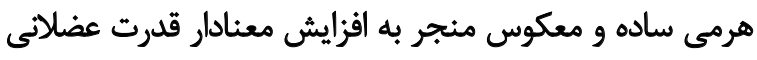

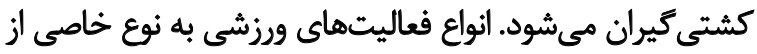

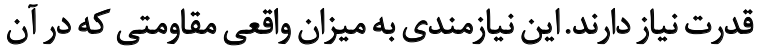

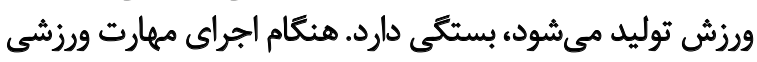

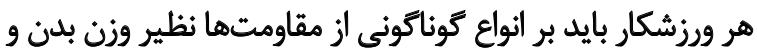

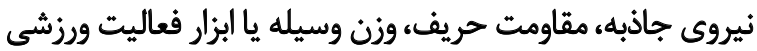

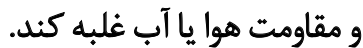

قدرت يكى از قابليتهاى جسمانى بايه است كه به واسطه فرايند

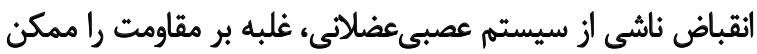

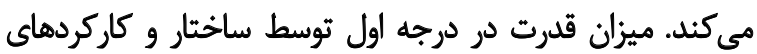

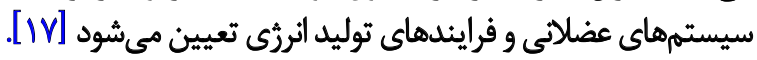

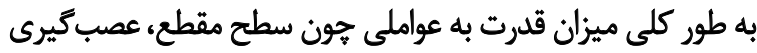

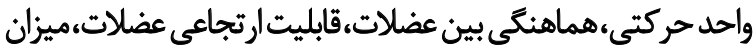
ذخيره انررثى و توده خالص بدن وابسته است عذاتيت

يكى از عوامل بسيار مؤثر در قدرت، حداكثر توده خالص بدنى

1. Lean Mass Body (LMB)
آنترويومتريك و نيمرخ مهارتى كشتى تهيران آزادكار بود. نتايج

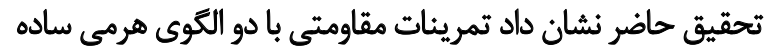

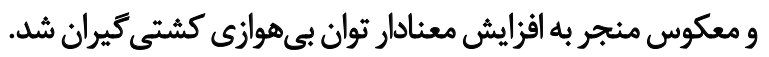
براى بسيارى از ورزشكاران، توانايى حفظ يك فعاليت شديد

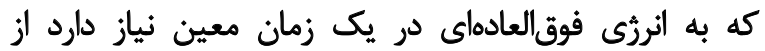

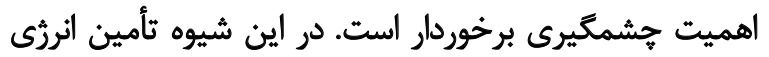

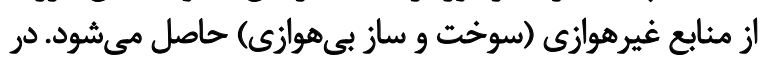

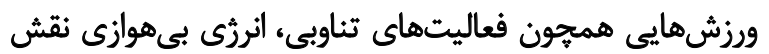

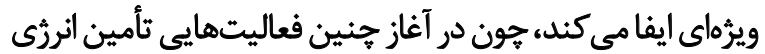

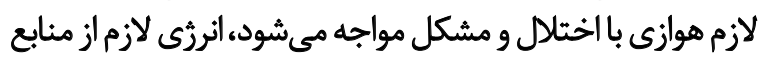
بي هوازى به دست مي آيد. مسيرهاي سوخت وساز كه در توليد بيىهوازي آدنوزين ترىفسفات

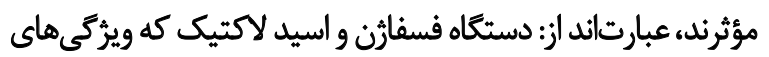

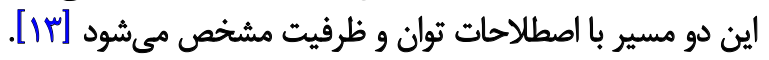

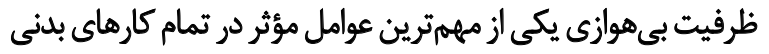

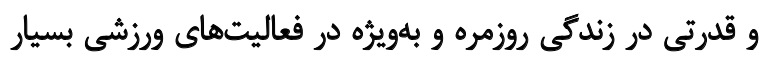

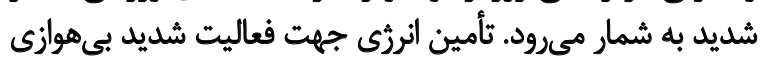

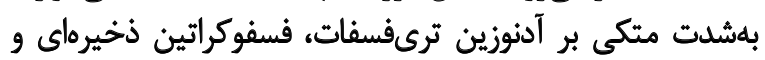
كليكوليز بيىهوازى است [ilf]

آدنوزين ترى فسفات، فسفوكراتين ذخيرهاي و مُليكوليز سريع،

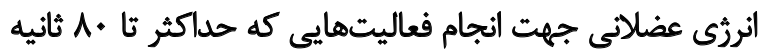

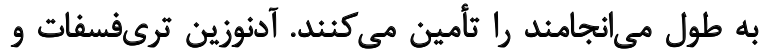

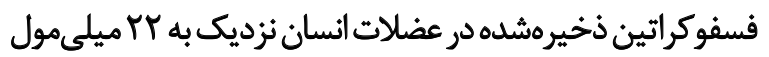

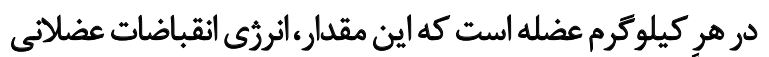

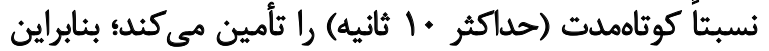




\section{نتيجلكيرى نهايی}

نتايج يُروهش حاضر نشان داد كه نمايه توده بدن، درصد جربى

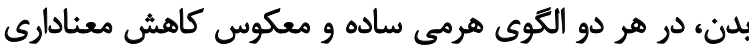

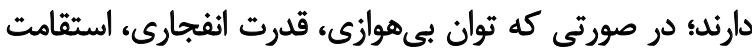

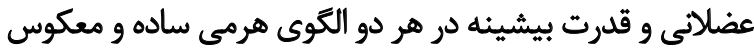

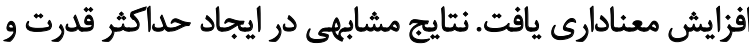

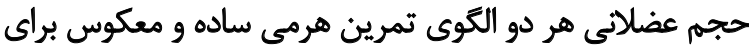

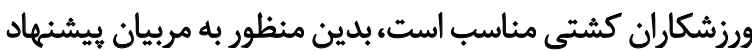

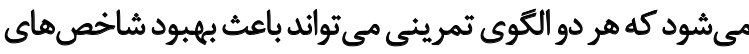
زيست حركتى كشتى

مالاحظات اخلاقى

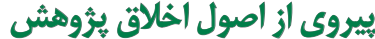

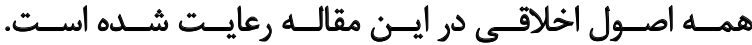

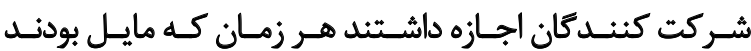

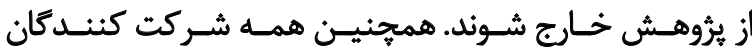

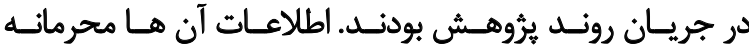

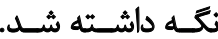

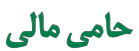
ايسن تحقيـق هيـج كونـهـ كمـك مالـ از سـازمانهاى تأميـن

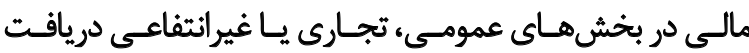

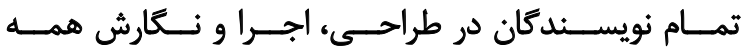

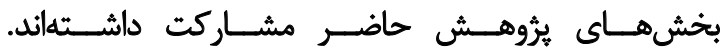

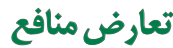

بنابر اظهار نويسندكان، اين مقاله تعارض منافع ندارد.

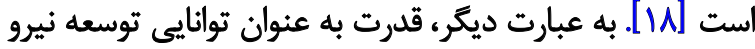

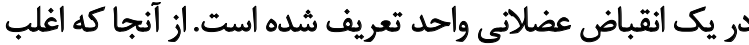

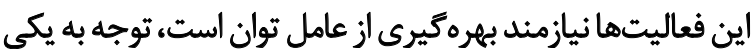

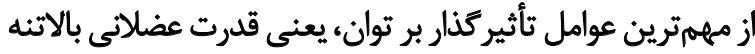

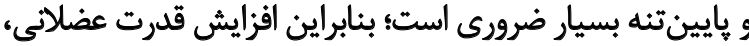

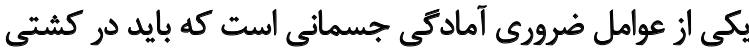

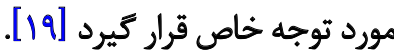
يكى از دلايل افزايش قدرت، سازگًارىهاى عصبى است كه در

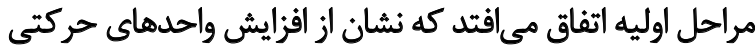

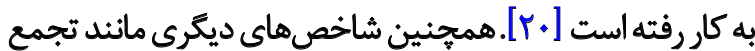

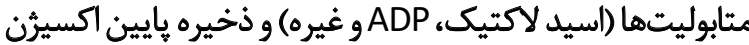

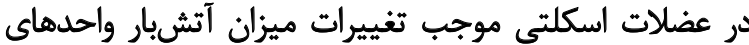

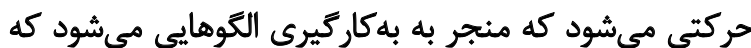

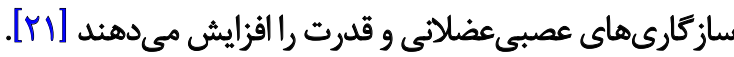
نتايج تحقيق حاضر نشان داد تمرينات مقاومتى بادو التوى

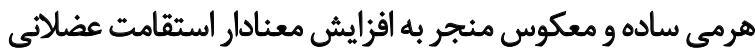

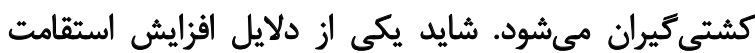

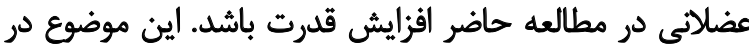

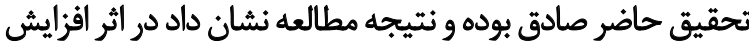

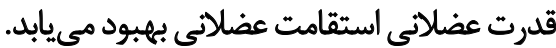

در اين زمينه، ولىيور دهنو و همكاران در مطالعه خود نشان

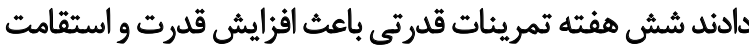

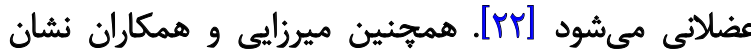

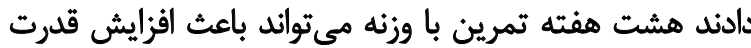

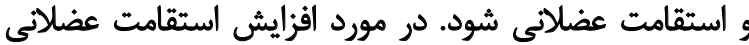

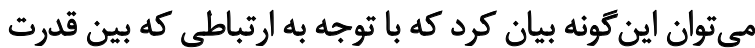

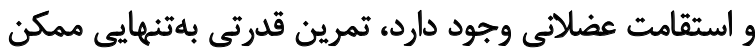

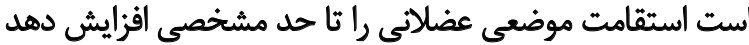

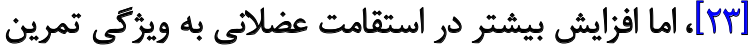

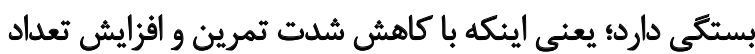

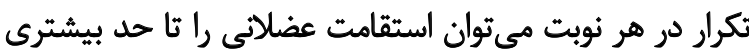

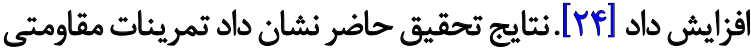

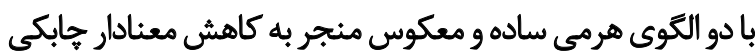

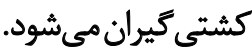

رواسى و همكاران با بررسى اثر هشت هفته تمرين تركيبى تئي

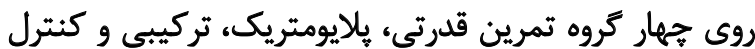

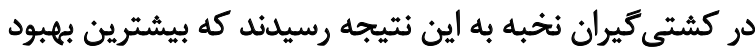

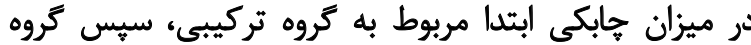

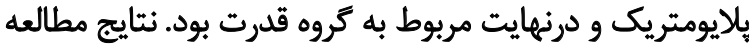

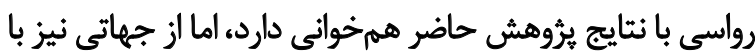

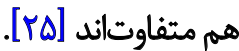




\section{References}

[1] Michaels GN. Arm wrestling training machine. Google Patents; 1988.

[2] Farzad B, Gharakhanlou R, Agha-Alinejad H, Curby DG, Bayati M, Bahraminejad $M$, et al. [Physiological and performance changes from the addition of a sprint interval program to wrestling training (Persian)]. J Strength Cond Res. 2011; 25(9):2392-9. [DOI:10.1519/ JSC.0b013e3181fb4a33] [PMID]

[3] Carpinelli R. Berger in retrospect: Effect of varied weight training programmes on strength. Br J Sports Med. 2002; 36(5):319-24. [DOI:10.1136/bjsm.36.5.319] [PMID] [PMCID]

[4] Häkkinen K. Neuromuscular and hormonal adaptations during strength and power training. A review. The Journal of sports medicine and physical fitness. 1989; 29(1):9-26. https://europepmc.org/article/ $\mathrm{med} / 2671501$

[5] Baechle TR, Earle RW. Weight training: Steps to success, New York: Human Kinetics. 2019. https://books.google.com/ books?id=DPB6DwAAQBAJ\&printsec

[6] Kraemer WJ, Gordon S, Fleck S, Marchitelli L, Mello R, Dziados J, et al. Endogenous anabolic hormonal and growth factor responses to heavy resistance exercise in males and females. Int J Sports Med. 1991; 12(02):228-35. [DOI:10.1055/s-2007-1024673] [PMID]

[7] Bompa T, Bompa TO, Carrera M. Periodization training for sports. Amsterdam: Elsevier; 2005.

[8] Mirzaei B, Arazi H, Curby D, Barbas I, Moghaddam MG, Hosseini Y. [The effects of two different resistive loading patterns on strength, hypertrophy, anaerobic power and endurance in young wrestlers (Persian)]. Int J Wrestl Sci. 2012; 2(1):41-7. [DOI:10.1080/21615667.2012.10878943]

[9] Häkkinen K, Kraemer WJ, Pakarinen A, Tripleltt-Mcbride T, McBride JM, Häkkinen $A$, et al. Effects of heavy resistance/power training on maximal strength, muscle morphology, and hormonal response patterns in 60-75-year-old men and women. Appl Physiol Nutr Metab. 2002; 27(3):213-31. [DOI:10.1139/h02-013] [PMID]

[10] Cronin JB, McNair PJ, Marshall RN. Is velocity-specific strength training important in improving functional performance? J Sports Med Phys Fitness. 2002; 42(3):267-73. [PMID]

[11] Mikesky AE, Mikesky KN, Getchell B. Physical Fitness: A way of life. Traverse City: Cooper Publishing Group; 2008.

[12] Bompa TO, Di Pasquale MG, Cornacchia L. Serious strength training. Human Kinetics; 2003. https://books.google.com/ books?id=3HNkMkJ9XOwC\&dq

[13] Hoff J, Helgerud J. Endurance and strength training for soccer players. Sports Med. 2004; 34(3):165-80. [DOI:10.2165/00007256-20043403000003] [PMID]

[14] Meckel Y, Machnai O, Eliakim A. Relationship among repeated sprint tests, aerobic fitness, and anaerobic fitness in elite adolescent soccer players. J Strength Cond Res. 2009; 23(1):163-9. [DOI:10.1519/ JSC.0b013e31818b9651] [PMID]

[15] Reilly T, Lees A, Davids K, Murphy W. Science and football (Routledge Revivals): Paper presented at the first World Congress of Science and Football, Liverpool, 13-17th April 1987. [DOI:10.4324/9780203720035]

[16] Plowman SA, Smith DL. Exercise physiology for health fitness and performance. San Francisco: Lippincott Williams \& Wilkins; 2013. https:// books.google.com/books?id=fYiqixSbhEAC\&printsec=frontcover\&dq
[17] Pareja-Blanco F, Rodríguez-Rosell D, Sánchez-Medina L, Gorostiaga E, González-Badillo J. Effect of movement velocity during resistance training on neuromuscular performance. Int J Sports Med. 2014 35(11):916-24. [DOI:10.1055/s-0033-1363985] [PMID]

[18] Nowacki P, Cai D, Buhl C, Krummelbein U. Biological performance of German soccer players (professionals and juniors) tested by special ergometry and treadmill methods. Sci Football. 1988; 1:145-57. https:// www.taylorfrancis.com/chapters/

[19] Öberg B, Möller M, Gillquist J, Ekstrand J. Isokinetic torque levels for knee extensors and knee flexors in soccer players. Int J Sports Med. 1986; 7(01):50-3. [DOI:10.1055/s-2008-1025735] [PMID]

[20] Al'Hazzaa H, Almuzaini K, Al-Refaee S, Sulaiman M. Aerobic and anaerobic power characteristics of Saudi elite soccer players. J Sport Med Phys Fit. 2001; 41(1):54-61. https://search.proquest.com/openview/749200 b790edd165f825f295a9a4126b/1?pq-origsite=gscholar \&cbl=4718

[21] Goto K, Ishii N, Kizuka T, Takamatsu K. The impact of metabolic stress on hormonal responses and muscular adaptations. Med Sci Sports Exerc. 2005; 37(6):955-63. https://europepmc.org/article/med/15947720

[22] Valipour dehnou V, Mansournejad H, Gharakhanlou R, Sharafi F. [Response of strength, power and muscle endurance to resistance training with different rest intervals (Persian)]. J Sport Biosci. 2015; 7(1):77-91. https://jsb.ut.ac.ir/mobile/article_54278.html?lang=en

[23] Mohebbi H, Sangdovini M, Mirzaei B. [Effect of two resistance training programs on muscular strengthin untrained men (Persian)]. Metab Exerc. 2012; 2(1):79-92. https://jme.guilan.ac.ir/article_675.html?lang=en

[24] Ratamess NA, Alvar BA, Evetoch TE, Housh TJ, Ben Kibler W, Kraemer WJ, et al. Progression models in resistance training for healthy adults. Med Sci Sports Exerc. 2009; 41(3):687-708. [DOI:10.1249/ MSS.0b013e3181915670] [PMID]

[25] Ravasi A, Gaeini A, Tasmeh M, Abdi H, Abdolmohammadi A. [The effect of plyometric, strength and complex training on agility of young free style wrestlers in Ilam City (Persian)]. J Sports Sci. 2014; 6(2):191-204. https:// jsb.ut.ac.ir/article_50862.html?lang=en 